BNL-113228-2016-JA

\title{
Nanoscale Origins of Ferroelastic Domain Wall Mobility in Ferroelectric Multilayers
}

\author{
Hsin-Hui Huang, Zijian Hong, Huolin L. Xin , Dong Su, \\ Long-Qing Chen, Guanzhong Huang, Paul R. Munroe and Nagarajan Valanoor
}

Submitted to ACS Nano

October 2016

Center for Functional Nanomaterials

Brookhaven National Laboratory

\author{
U.S. Department of Energy \\ USDOE Office of Science (SC), \\ Basic Energy Sciences (SC-22)
}

Notice: This manuscript has been authored by employees of Brookhaven Science Associates, LLC under Contract No. DE-SC0012704 with the U.S. Department of Energy. The publisher by accepting the manuscript for publication acknowledges that the United States Government retains a non-exclusive, paid-up, irrevocable, world-wide license to publish or reproduce the published form of this manuscript, or allow others to do so, for United States Government purposes. 


\section{DISCLAIMER}

This report was prepared as an account of work sponsored by an agency of the United States Government. Neither the United States Government nor any agency thereof, nor any of their employees, nor any of their contractors, subcontractors, or their employees, makes any warranty, express or implied, or assumes any legal liability or responsibility for the accuracy, completeness, or any third party's use or the results of such use of any information, apparatus, product, or process disclosed, or represents that its use would not infringe privately owned rights. Reference herein to any specific commercial product, process, or service by trade name, trademark, manufacturer, or otherwise, does not necessarily constitute or imply its endorsement, recommendation, or favoring by the United States Government or any agency thereof or its contractors or subcontractors. The views and opinions of authors expressed herein do not necessarily state or reflect those of the United States Government or any agency thereof. 


\section{Nanoscale Origins of Ferroelastic Domain Wall}

\section{Mobility in Ferroelectric Multilayers}

Hsin-Hui Huang ${ }^{1}$, Zijian Hong ${ }^{2} \ddagger$, Huolin L. Xin ${ }^{3} \neq$, Dong Su ${ }^{3}$, Long-Qing Chen ${ }^{2}$, Guanzhong Huang ${ }^{3,4}$, Paul R. Munroe and Nagarajan Valanoor $^{1 *}$

${ }^{1}$ School of Materials Science and Engineering, University of New South Wales, Sydney, NSW, 2052, Australia

${ }^{2}$ Department of Materials Science and Engineering, Pennsylvania State University, University Park, Pennsylvania, 16802-5006, USA

${ }^{3}$ Center for Functional Nanomaterials, Brookhaven National Laboratory, Upton, Brookhaven, New York, 11973, USA

${ }^{4}$ Department of Materials Science and Engineering, Stony Brook University, Stony Brook, New York, 11794-3400, USA

\section{Received Date:}

*Corresponding author: nagarajan@unsw.edu.au

† These authors contributed equally 


\section{ABSTRACT}

The nanoscale origins of ferroelastic domain wall motion in ferroelectric multilayer thin films that lead to giant electromechanical responses are investigated. We present direct evidence for complex underpinning factors that result in ferroelastic domain wall mobility using a combination of atomic-level aberration corrected scanning transmission electron microscopy and phase-field simulations in model epitaxial (001) tetragonal ( $\mathrm{T}$ ) $\mathrm{PbZr}_{\mathrm{x}} \mathrm{Ti}_{1-\mathrm{x}} \mathrm{O}_{3}$ $(\mathrm{PZT}) /$ rhombohedral $(\mathrm{R}) \mathrm{PbZr}_{\mathrm{x}} \mathrm{Ti}_{1-\mathrm{x}} \mathrm{O}_{3}(\mathrm{PZT})$ bilayer heterostructures. The local electric dipole distribution is imaged on an atomic scale for a ferroelastic domain wall that nucleates in the Rlayer and cuts through the composition breaking the $\mathrm{T} / \mathrm{R}$ interface. Our studies reveal a highly complex polarization rotation domain structure that is nearly on the knife-edge at the vicinity of this wall. Induced phases, namely tetragonal-like and rhombohedral-like monoclinic were observed close to the interface, and exotic domain arrangements, such as a half-four-fold closure structure, are observed. Phase field simulations show this is due to the minimization of the excessive elastic and electrostatic energies driven by the enormous strain gradient present at the location of the ferroelastic domain walls. Thus, in response to an applied stimulus, such as an electric field, any polarization reorientation must minimize the elastic and electrostatic discontinuities due to this strain gradient, which would induce a dramatic rearrangement of the domain structure. This insight into the origins of ferroelastic domain wall motion will allow researchers to better "craft" such multilayered ferroelectric systems with precisely tailored domain wall functionality and enhanced sensitivity, which can be exploited for the next generation of integrated piezoelectric technologies. 


\section{KEYWORDS}

Nanoscale ferroelectrics, epitaxial thin films, ferroelastic domains, aberration-corrected scanning transmission electron microscopy, nanoscale elelctromechanics 
The precise level of atomic control made possible by modern thin-film growth techniques has led to a number of interface-driven breakthroughs in epitaxial ferroelectric thin films. ${ }^{1-5}$ In particular, multilayered ferroelectric heterostructures, which possess an intentionally created chemical composition gradient along the growth direction, have recently attracted significant attention due to their enhanced polarization ${ }^{6}$ and electromechanical properties. ${ }^{7-10}$ This purposely induced chemical gradient results in enhanced pyroelectric, ${ }^{9,11}$ piezoelectric, ${ }^{12-14}$ and dielectric responses, ${ }^{10,15}$ some several times that of a single-layer counterpart. The realization of high- performance electromechanical thin films is invaluable for advanced nanoelectronic technologies such as piezoelectric energy harvesting, ${ }^{16}$ ultra-sensitive medical imaging, ${ }^{17}$ motorless lenses ${ }^{18}$ etc. A distinct feature of such multilayers, irrespective of either the number layers ${ }^{19}$ or the morphological nature of the gradient, i.e. whether superlattice-like ${ }^{10}$ or exhibiting a continuous composition gradient, ${ }^{14}$ is that the enhanced electromechanical responses come from extrinsic contributions, predominantly from ferroeleastic domain wall motion. Although there is enough direct evidence that confirms this, ${ }^{10,13-14}$ why this should indeed be the case is not so clear. A clear understanding of the origins of this lability will allow researchers to better "craft" such multilayered ferroelectric systems with precisely tailored domain wall functionality ${ }^{20}$ and enhanced sensitivity. ${ }^{21}$ This will open unexplored paradigms, in which ferroelastic domain wall engineered ferroelectric thin films $\mathrm{s}^{20,22-23}$ can be exploited, for the next generation of integrated piezoelectric technologies, particularly those that demand ultra-high performance for relatively low input energies for power efficiencies.

Conventionally, it is thought that substrate-induced clamping in thin films is strong enough to prevent any ferroelastic domain wall motion. ${ }^{24-26}$ Although layering could relieve this clamping to some extent (depending on the thickness and elastic properties of the ferroelectric layers and the substrate) the answer does not lie in the mechanical relief alone. The electric field-induced coupling and electrostatic interactions between adjacent layers due to the forced 
proximity and two-dimensional constraint is equally important, ${ }^{27-28}$ even more so at the composition-breaking interfaces where the energetic cost to maintain the polarization must be very high. Finally, the compositional gradient induces significant local change in strain, and hence one must also account for flexoelectricity at the nanoscale. ${ }^{29-30}$ Since the width of the ferroelastic domain wall is typically only a few unit cells, ${ }^{31-33}$ the mechanisms that govern its dynamic response must be at such length scales, which means a nanoscale level of understanding is ideally desired.

Here, we reveal some of the complex underpinning factors that lead to the observed domain wall mobility by studying the local electric dipole distribution on an atomic scale near a composition-breaking interface for epitaxial (001) tetragonal (T) $\mathrm{PbZr}_{\mathrm{x}} \mathrm{Ti}_{1-\mathrm{x}} \mathrm{O}_{3}$ $(\mathrm{PZT}) /$ rhombohedral $(\mathrm{R}) \mathrm{PbZr}_{\mathrm{x}} \mathrm{Ti}_{1-\mathrm{x}} \mathrm{O}_{3}(\mathrm{PZT})$ bilayer heterostructures. By exploiting recent advances in spherical-aberration corrected scanning transmission electron microscope (Cscorrected STEM) imaging techniques, ${ }^{34-38}$ we map the magnitude and direction of polarization displacement at an atomic level. The Cs-corrected approach has previously been successfully applied to understand a number of critical nanoscale ferroelectric phenomena such as size effects, ${ }^{39-40}$ topological structures ${ }^{41}$ and domain switching, ${ }^{42-43}$ particularly in those ferroelectric perovskites with a strong strain-polarization coupling. In addition, the electronic structure and composition of perovskite oxide heterointerfaces can be accurately mapped at sub-angstrom resolution ${ }^{44-46}$ using Cs-corrected STEM-based spectroscopy methods, allowing the accurate mapping and location of interfaces. These capabilities are exploited here to show that the formation of ferroelastic domains in one layer (in our particular case the PZT (T) layer) leads to an enormous local strain gradient at the vicinity of the domain wall. ${ }^{47}$ The magnitude of this strain gradient is high enough to locally rotate the polarizations in the $\mathrm{T}$ and $\mathrm{R}$ layers. Indeed induced phases, namely a tetragonal-like monoclinic in the T-layer and rhombohedrallike monoclinic in the R-layer are observed close to the interface, which then make it possible 
to achieve complex domain topologies such as a half four-fold closure. Phase field simulations find that this complex domain arrangement is driven by minimization of the excessive elastic and electrostatic energies due to the strain gradient near/at the interface. As such, this domain arrangement is on a knife-edge teetering between several competing polar arrangements, and thus any applied field that switches one component of the polarization results in a massive upheaval of the entire equilibrium domain state.

\section{RESULTS AND DISCUSSION}

The ferroelastic domain structure of the bilayer PZT (T)/ PZT (R) layer is shown in the high angle annular dark field scanning transmission electron microscopy (HAADF-STEM) images, where Figure 1 (a) illustrates the top PZT (T) layer and Figure 1 (d) shows the bottom PZT (R) layer. The choice of this thin film bilayer is driven by prior observations of robust and reliable ferroelastic domain wall mobility in this system. ${ }^{13}$ Synchrotron x-ray diffraction, coupled with conventional bright field TEM analysis, confirmed the crystal structure of the PZT (R) to be rhombohedral with average in-plane and out-of-plane lattice parameters of $\sim 0.410 \mathrm{~nm} .{ }^{48}$ On the other hand, the PZT $(\mathrm{T})$ layer has a tetragonal $c / a$ polydomain structure with average lattice parameters of $\mathrm{c}=0.415 \mathrm{~nm}$ and $\mathrm{a}=0.398 \mathrm{~nm}$. (The domain wall separating the " $c$ " and " $a$ " axis oriented domains is typically referred to as a $90^{\circ}$ domain wall and we will follow this nomenclature). Both PZT layers are fully relaxed on a macroscale by the formation of threading dislocations in the PZT (R) layer and by the formation of $90^{\circ}$ polydomain structures in the $\mathrm{PZT}$ (T) layer, respectively. The existence of the polydomain structure is further confirmed using geometric phase analysis (GPA) as described in the Supporting Information S1.

The insets are magnified images overlying the $c$ - and $a$-domain regions. Each of them is depicted schematically in Figure 1 (b) and (c). The pink circles denote $\mathrm{Pb}^{2+}$ columns and the blue circles denote $\mathrm{Ti}^{4+}$ or $\mathrm{Zr}^{4+}$ columns. It is noted that the $\mathrm{O}^{2-}$ columns are not visible due to 
the weak scattering factor of the electrons. ${ }^{49}$ Nonetheless, when the atom arrangement of the PZT (T) layer exhibits a shift of the central $\mathrm{Zr}^{4+} / \mathrm{Ti}^{4+}$ cations, for example, in $a$-domains, the central $\mathrm{Zr}^{4+} / \mathrm{Ti}^{4+}$ cation moves toward the [010] direction, the $\mathrm{O}^{2-}$ columns are shifted much more strongly. As a consequence, a charge dipole forms due to the separation of the negative $\left(\mathrm{O}^{2-}\right)$ and positive $\left(\mathrm{Ti}^{4+} / \mathrm{Zr}^{4+}\right.$ and $\left.\mathrm{Pb}^{2+}\right)$ charges. Thus, the domain structure over this area corresponds to tetragonal $a$-domain with the direction of the spontaneous polarization pointing toward the $[0 \overline{1} 0]$ direction. Even though the schematic diagram of structural distortion is exaggerated, the atomic shifting of the PZT is $\sim 30 \mathrm{pm}$ as observed by Jia et al..$^{34,50}$ and is well within the resolution of an aberration (Cs)-corrected microscope. The atomic resolution image of the PZT (R) layer near the LSMO layer is shown in Figure $1(\mathrm{~d})$. The central cation $\mathrm{Ti}^{4+} / \mathrm{Zr}^{4+}$ is shifted along the $[0 \overline{1} \overline{1}]$ diagonal axis of the unit cell. Given that the PZT $(\mathrm{R})$ layer has a rhombohedral structure with $<111>$ polarizations, in a (001) oriented system, this can result in parallel stripes rotating $71^{\circ}$ or $109^{\circ}$ along the in-plane direction. ${ }^{51}$ However, determination of the polarization direction in the rhombohedral system is much more complicated than in the tetragonal system. ${ }^{52}$ Here, due to the limitation with respect to acquisition orientation, it is assumed that the observed atomic displacement in Figure $1(\mathrm{~d})$ is along either the $[1 \overline{1} \overline{1}]$ or $[\overline{1} \overline{1} \overline{1}]$ directions, as schematically illustrated in Figure 1 (e).

It is noted that the position of the $c_{-}, a-$, and rhombohedral domains were identified based on local strain mapping using geometric phase analysis (GPA) analysis, as addressed in Supporting Information Figure S2 and Figure S3. Figure S2 also reveals the presence of both "sharp" and "broadened" ferroelastic domain walls. This broadening of domain walls may be due to its coupling with local point defects or inhomogeneity in the local distortion. It was shown that the coupling of the ferroelastic domain wall with point defects can lead to a giant electromechanical response $\mathrm{e}^{53}$ and atypical domain configurations including charged $90^{\circ}$ domain walls. ${ }^{54}$ Therefore, the broadened domain wall observed here not only accommodates 
local strains and mobile point defects, but also could play a significant role in the electromechanical response.

Having characterized and ascertained that the method has the capacity to atomically resolve the domain structure of the bilayer system, it is necessary to locate the interfacial boundary precisely. An electron energy-loss spectroscopy (EELS) line scan was applied along the outof-plane direction from point 1 to point 6 marked in the EELS-STEM image shown in the Supporting Information Figure S4 (a). This was acquired by moving the STEM probe serially along the line, and a series of EEL spectra were recorded at each point. EEL spectra for the Ti$\mathrm{L}_{2,3}$ edges were extracted and plotted in Figure S4 (b). The normalized intensity profile (Figure S4 (c)) for the Ti shows a stronger signal from point 1 to point 3 . On the other hand, a weaker signal is observed at point 4 to point 6 . According to the deposition conditions, there is more Ti in the PZT (T) $\left(\mathrm{PbZr}_{0.30} \mathrm{Ti}_{0.70} \mathrm{O}_{3}\right)$ layer compared to the PZT (R) $\left(\mathrm{PbZr}_{0.55} \mathrm{Ti}_{0.45} \mathrm{O}_{3}\right)$ layer. Therefore, it is confirmed that the interfacial boundary of the two layers can be identified as allocated in between point 3 and point 4 . It should be noted that the EELS line scans were applied each time before imaging to determine the location of the interface. We are now in a position to correlate the unit cell displacement vectors with respect to the lattice of the individual constituents of bilayer. 


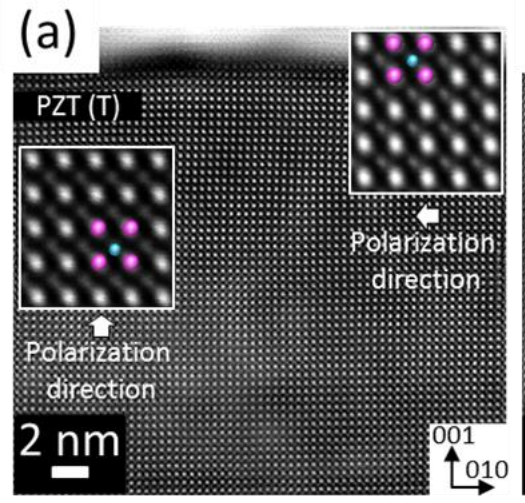

(c)

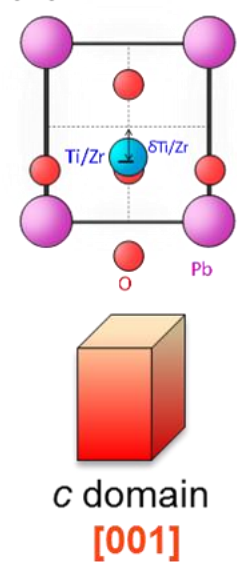

(d)
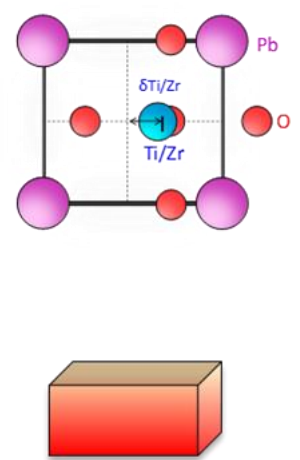

$a_{1}$ domain

[010]

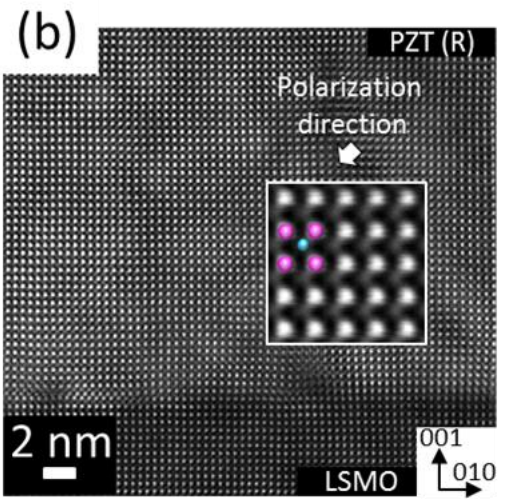

(e)
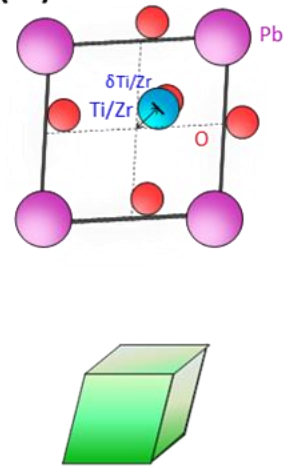

$R$ domain [111] polarization

Figure 1. (a) Aberration-corrected HAADF image of the PZT (T) layer near the outer surface of the sample. The insets are high magnification images of the overlying areas. It can be seen that there is a subtle change in the atomic column position. The central cation, $\mathrm{Ti} / \mathrm{Zr}$, was shifted rightwards along the [001] or [010] direction, as schematically illustrated in (b) and c). These correspond to the tetragonal c- and a- domains, respectively. Meanwhile, a spontaneous polarization is generated in the opposite direction. (d) Aberration-corrected HAADF image showing the PZT $(\mathrm{R})$ layer taken near the PZT (R)/ LSMO interface. The inset is an enlarged image of the overlying region. The corresponding schematic illustration is illustrated in (e), showing the Ti/Zr cation displaced along either the $[1 \overline{1} \overline{1}]$ or $[\overline{1} \overline{1} \overline{1}]$ directions.

The displacement vectors of each unit cell were mapped and superimposed on the HAADF image, as shown in Figure 2 (a) in the vicinity of a $c / a\left(90^{\circ}\right)$ domain found across the interface. 
The raw image without the vectors and its corresponding drift and noise-filtered image are shown in Figure S5-1. Before going to the complex interfacial case of Figure 2, the displacement vectors for only the T-layer and the R-layer were first mapped to ensure reliable calibration. Details of the mapping process can be found in the Supporting Information, Figure S5-2 and results can be found in S6. Note that the raw images for only the R-layer and only the T-layer (that is far away from the interface) were able to not only successfully map the polarization, but also yield the displacement direction that is crystallographically correct (see S6). This gives confidence that the quality of the STEM images is high enough for further analysis. It is noted that the displacement vector was measured from the centre of the unit cell toward the actual atomic column position, as indicated by blue arrows. In other words, the corresponding spontaneous polarization $\left(\mathrm{P}_{\mathrm{s}}\right)$ is pointing in the opposite direction of the lattice displacement vector, as shown by the bolder white arrows. The interfacial boundary was determined by electron energy loss spectroscopy (EELS) (as mentioned in the Supporting Information S4) and marked by a white dashed line. The viewing direction is along [100] direction. By careful inspection, there are four different polarization directions in the PZT (T)

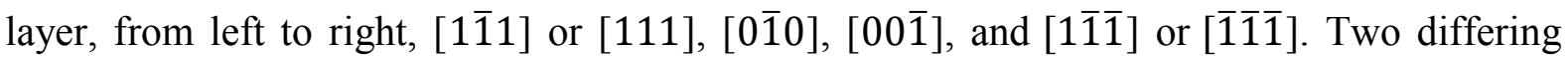
orientations can be discerned by detailed analysis on the magnitude and the tilting angle, or alternately analysis by electron holography techniques. ${ }^{55}$ Even more intriguingly in the PZT (R) layer, there are three different types of spontaneous polarizations, from left to right, [11 $\overline{1}]$ or $[1 \overline{1} \overline{1}],[00 \overline{1}]$, and $[0 \overline{1} 0]$. This is in stark contrast to regions that are further away from the interface. In S6 (Supporting Information), we plot the polarization displacement vector in the "bulk" of the R-layer, which confirms a uniform polarization direction along $<111>$. Thus, we find considerable polarization rotation in both layers close to the interface.

The presence of polarization rotation at a ferroelastic domain wall was previously observed for $\mathrm{PbTiO}_{3}$ films grown on $\mathrm{DyScO}_{3}$ substrates. ${ }^{47}$ Therefore, the presence of the [1]1] or [111], 
and $[1 \overline{1} \overline{1}]$ or $[\overline{1} \overline{1} \overline{1}]$ polarization directions for the nominally tetragonal PZT layer and, $[00 \overline{1}]$ and $[0 \overline{1} 0]$ polarizations for the nominally rhombohedral PZT layer, is a strong indicator of the presence of a $90^{\circ}$ domain wall. This $90^{\circ}$ domain is found across the interface, along with the $[00 \overline{1}]$ and $[0 \overline{1} 0]$ directions. The domain wall boundary, determined directly by mapping the change in $\mathrm{Ti} / \mathrm{Zr}$ atomic displacement, ${ }^{38,56}$ is identified with a "head-to-tail" arrangement of the spontaneous polarization. Such a head-tail coupled arrangement leads to an uncharged domain wall (UCDW), as indicated in the corresponding schematic in Figure 2 (b). It is intriguing to note that this $90^{\circ}$ domain terminates within the PZT (R) matrix, thus explaining why rotation was also observed in the PZT (R) layer. A closure-like polar arrangement is found at the point where the $90^{\circ}$ domain is terminated in the PZT (T) layer, as indicated by the red arrows in Figure 2 (b). The continuous rotation of the dipole directions from the diagonal-axis direction pointing up along the $90^{\circ}$ domain wall (right) to the diagonal-axis direction pointing down along the $90^{\circ}$ domain wall (left) hints at some form of chirality. The presence of this domain wall chirality was first reported by Houchmandzadeh et al. ${ }^{57}$ stating that chirality will be generated if there is more than one parameter involved. Notably, this is not like the typical fourfold closure structure with four continuous polarization vectors at $90^{\circ}$ converging into a central vertex, as schematically sketched in Figure 2 (c) Left. As an alternative, these domain structures behave like a half fourfold closure (Figure 2 (d) Right), as predicted by Srolovitz and Scott. ${ }^{58}$ Such closures have been previously observed for $\mathrm{PbZr}_{0.20} \mathrm{Ti}_{0.80} \mathrm{O}_{3} / \mathrm{SrTiO}_{3}$ thin films ${ }^{50}$ and for vortex-like nanodomain arrays at $109^{\circ}$ domain walls at interfaces in $\mathrm{BiFeO}_{3}$ films. ${ }^{52}$ Note that in both previous instances, the closure domains occur at a source of a nonuniform electric field formed under external stimulus, ${ }^{59-60}$ induced by charge compensation at domain boundaries ${ }^{52,61}$ or in nanodots. ${ }^{62-63}$ In the present study, the needle-like morphology of the ferroelastic domain would be an excellent source for such a non-uniform electric field, as confirmed by the phase field simulations (discussed later). We observed such local polarization 
rotation close to the $\mathrm{R} / \mathrm{T}$ interface as a general phenomenon, particularly both the displacements and the tetragonality displaying strong shear distortions.

In order to sustain the magnitudes of polarization rotation shown in Figure 2 (a), there must be large local elastic gradients along both normal and horizontal directions, i.e. the strain gradient cannot be neglected. It has now been convincingly shown that the shear component that arises from the flexoelectric tensor is strong enough in these ferroelectrics to create polarization rotation, ${ }^{47,}{ }^{64-66}$ and the flexoelectric effect is amplified at nanoscale. ${ }^{67}$

(a)

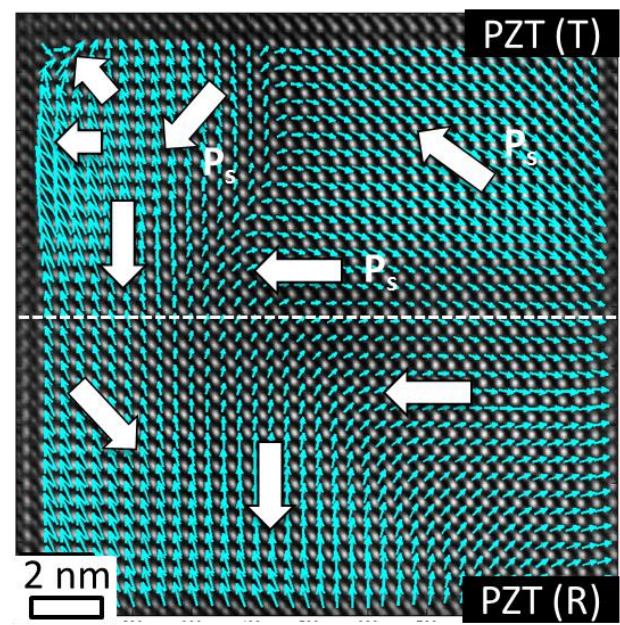

(b)

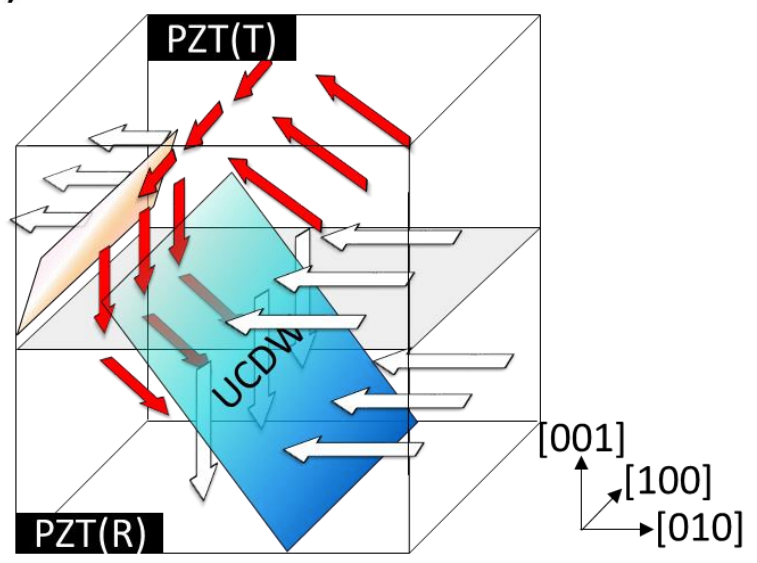

(c)

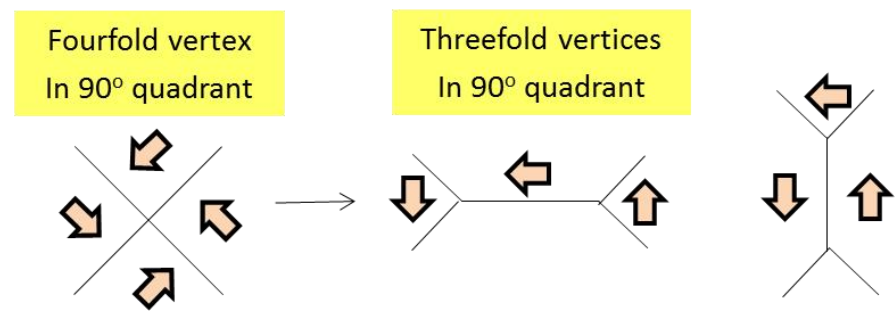

Figure 2. (a) Superposition of HAADF-STEM images and Ti4 $+/ \mathrm{Zr} 4+$ displacement vector maps showing a complex feature near the interface. The arrows denote the direction of the Ti4+/Zr4+ displacement directions. The dashed line indicates the PZT (T)/PZT (R) interface. (b) Illustration of intersection of domains at/near the T/R interface in (a). The white arrows are the spontaneous polarizations of the domains along the $[\mathbf{0} \overline{\mathbf{1}} \mathbf{0}]$ and $[\mathbf{0 0} \overline{\mathbf{1}}]$ directions. The red 
arrows depict a closure-like polar configuration, which is similar to the half fourfold closure as schematically illustrated in (c).

This is indeed confirmed by the corresponding tetragonality map (ratio of out-of-plane to inplane lattice parameters or c/a) of the region from Figure 2, as shown in Figure 3 (a), where the tetragonality value is indicated by colour scale bar. These were obtained by measuring the distance between pairs of the nearest neighbour $\mathrm{Pb}^{2+}$ atomic columns along the [001] and [010] directions. ${ }^{68}$ One observes immediately a stark inhomogeneity in tetragonality (as shown by the strong changes in colour) along/across the PZT (T)/PZT (R) interface - this is very different to the tetragonality map acquired from regions well-inside the individual $\mathrm{R}$ and $\mathrm{T}$ layers (see Figure S6-1 and S6-2 in the Supporting Information). Note that there are pockets of tetragonality larger than 1 adjacent to the interface, but as we move gradually away the tetragonality is either close to, or less than 1 , denoted by the predominantly blue contrast. The drop and variation across a few unit cells along the thickness direction indicates the presence of strong horizontal and vertical strain gradients. The effect of such large strain gradients can be found on the morphology (orientation and magnitude) of the polarization displacement vector. The polarization rotation map and the magnitude plots, acquired from the areas marked by the dashed boxes (from left to right, respectively) in Figure 3 (a), are shown in Figure 3 (b)(c)(d), (upper parts are polarization rotation and lower parts are magnitude). The rotation maps depict the degree of the polarization rotated from the interface ( 0 degree pointing to the left), whilst the magnitude map shows the amount of atomic displacement. It ranges from 0 to $\sim 23 \mathrm{pm}$, as revealed by the scale bar. This is in line with expectations for this composition of PZT (note that for $\mathrm{PbZr}_{0.2} \mathrm{Ti}_{0.8} \mathrm{O}_{3}$, a composition with larger spontaneous polarization, $c$-axis oriented films showed a displacement of $\sim 30 \mathrm{pm}) .{ }^{34,44}$ 
(a)

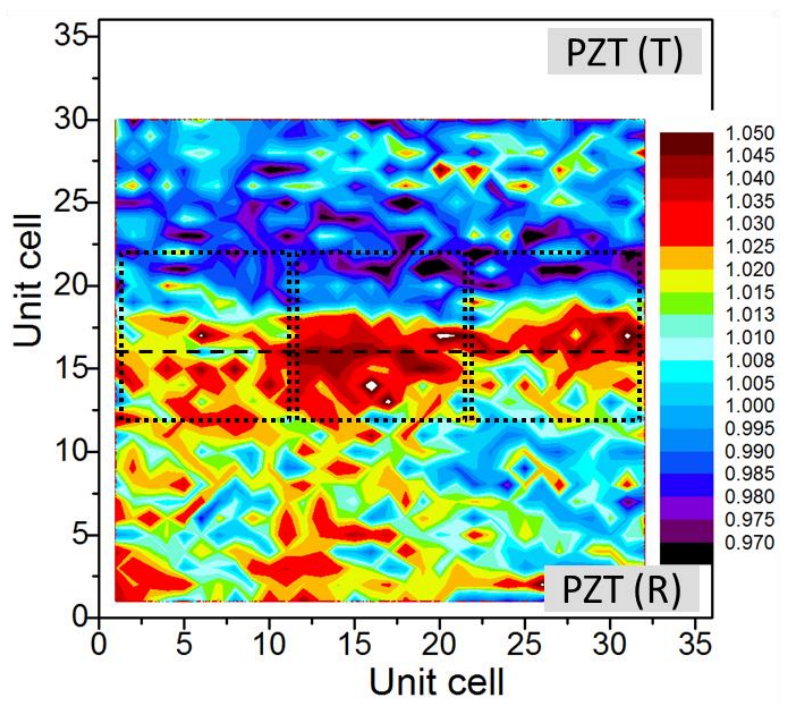

(b)

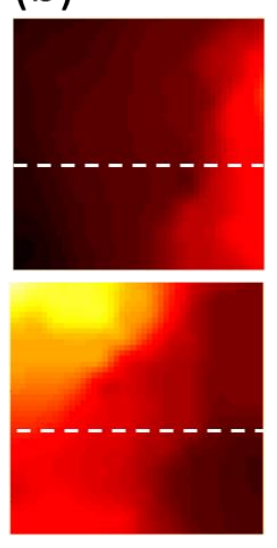

(c)

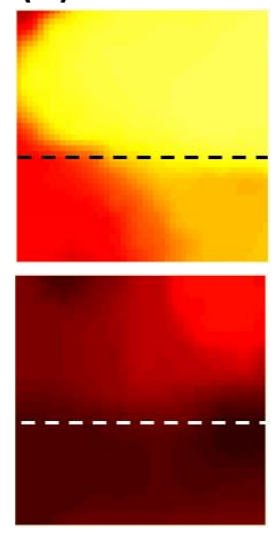

(d)

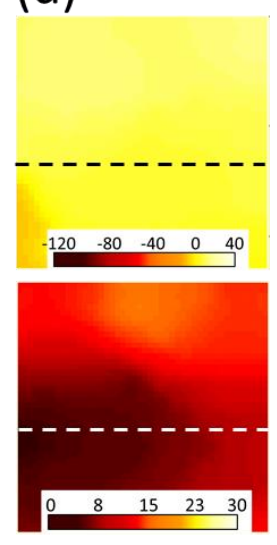

Figure 3. (a) The c/a ratio map (tetragonality map) of Figure 2 (a). Note that, inhomogeneousity in the c/a ratio is observed. (b)(c)(d) The polarization rotation map (upper) and the magnitude plots (lower), acquired from the areas marked by the dashed boxes (from left to right).

We start the analysis with Figure 3 (b). The corresponding region in Figure 2 reveals the polarization is predominantly downwards along the $c$-axis close to the interface, but $\sim 9$ unit cells away the polarization vector is tilted. This is indeed confirmed by the angle map (most of Figure 3 (b) is at $-90^{\circ}$, except at the right margins and top left where we begin to see the angle deviate due to the presence of a $90^{\circ}$ domain wall. As the region with the $-90^{\circ}$ polarization orientations are fully $c$-axis oriented they are expected to have tetragonality values typically $\sim 1.04$ and the largest atomic displacements. The map of Figure 3 (b) confirms this and, thus, it gives us confidence that the further measurements are not distorted by any scanning artefacts.

Most interesting is the case for Figure 3 (c) and 3 (d). Note that the polarization vector for both these regions is strongly tilted $\left(\sim 50^{\circ}\right)$ with the atomic displacements ranging from 8 to 15 pm. This means the lower tetragonalities observed in the corresponding regions from the map in Figure 3 (a) cannot stem from the simple notion that these are merely $a$-axis oriented regions 
(because if it were, we would expect to see rotation angles of $0^{\circ} / 180^{\circ}$ and atomic displacement of $\sim 23 \mathrm{pm}$ ). Rather it is from polarization canting, that is, the internal symmetry of a nominally tetragonal PZT layer is broken and reduced in the vicinity of the ferroelastic domain wall due to the strong polarization rotation driven by the flexoelectric effects. Such a reduction of internal symmetry due to polarization discontinuity was indeed expected in ref, ${ }^{47}$ but the authors were unable to directly visualize this for a number of reasons. In ultra-thin bilayers the tensile strain due to the underlying R-layer, coupled with the electrostatic minimization, can induce polarization rotation in the T-layer. ${ }^{69}$ Here we provide the direct experimental evidence of such phenomena. A similar trend can be found in Figure 3 (d) as well where there is polarization rotation along the vertical direction, whilst the tetragonality alters from low at the top layer to high at the bottom layer. Thus, there are pockets of interfacial regions that are neither (bulk) tetragonal nor the rhombohedral phase.

To elucidate the change of the internal symmetry of the domain structure at/near the interface, we combine the results from Figure 2 (a) and Figure $3(a)(b)(c)(d)$ to draw a schematic of the $\mathrm{T} / \mathrm{R}$ interface across a region 8 unit cells height and 32 unit cells wide, (Figure 4 (a)). According to the tetragonality and polarization direction, we categorize three types of structure, in-plane tetragonal-like monoclinic (blue), out-of-plane tetragonal-like monoclinic (yellow), and rhombohedral-like monoclinic (green). The out-of-plane tetragonal-like monoclinic appears in the right corner of the image, which has an out-of-plane tetragonal structure with tilted polarization pointing at the direction between the $<100>$ and $<111>$, as shown in the schematic in Figure 4 (b). On the other hand, the rhombohedral structure with polarization rotated away from the $<111>$ direction is characterized as rhombohedral-like monoclinic (Figure 4 (c)). 
(a)

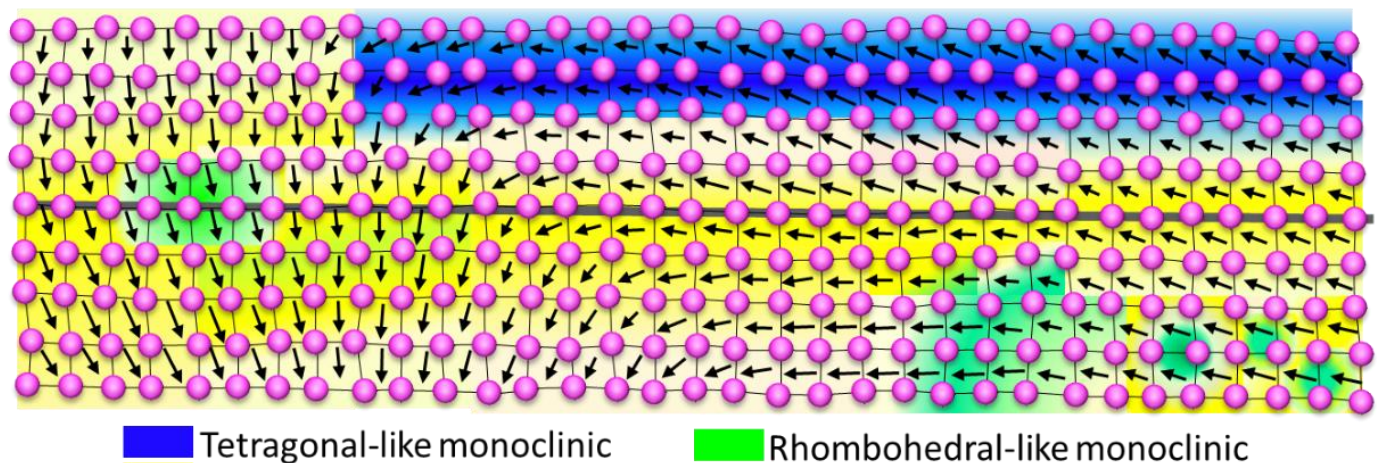

(b)

(c)
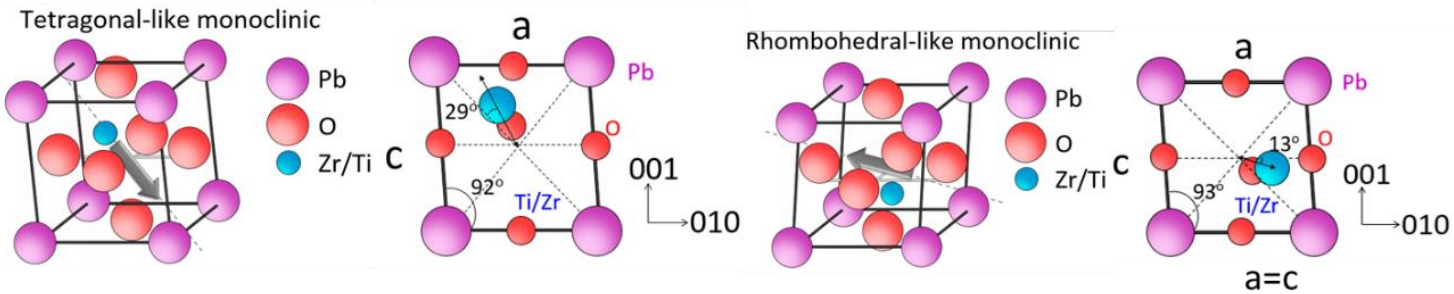

Figure 4. (a) Schematically illustration of the $T / R$ interface shows tetragonal-like monoclinic and rhombohedral-like monoclinic phases (middle). The arrow shows the polarization direction of each unit cell. Schematic of the (b) tetragonal-like and (c) rhombohedral-like monoclinic phases.

Having found direct experimental confirmation of rhombohedral-like monoclinic and tetragonal-like monoclinic domains at/near the T/R interface, the next step is to understand their origin and role. These rotational polar phases could be (i) formed to accommodate the large strain gradients between the two adjacent layers with (ii) the orientations driven so as to satisfy the electrical boundary conditions. To test our hypothesis, we perform phase-field calculations to simulate the domain structure in the PZT T/R bilayer, following the formalism by Xue et al. ${ }^{28}$ and Li et al. ${ }^{70}$ The domain structure of the as-grown film is shown in Figure 5 (a). It can be seen that the $\mathrm{T}$ layer is forming a classic ferroelastic/ferroelectric twin structure, with both 90 -degree $a / c$ and $a_{1} / a_{2}$ domain walls, as well as 180 -degree $c^{+} / c^{-}$and $a^{+} / a^{-}$domain 
walls. Typically, the 90-degree domain wall is primarily 45-degree oriented to form head-totail non-charged walls. Meanwhile, the R-twin structure is formed in the PZT (R) layer, with both 109 and 71-degree domain walls. The $a$-domains become wedge-like in shape near the interface, which penetrate through the interface up to several $\mathrm{nm}$, before being terminated by the PZT (R) domains (as observed experimentally). These wedge-like $a$-domains could cause large perturbations in the strain, thus, leading to strain gradients. Penetration of the PZT (R) domains into the T layer is also observed close to the interface, as initially observed by Xue et $a l .^{28}$

In Figure 5 (b) and (c), we plot the local polarization profile in the region where the $a$-domain lies. Figure 5 (b) shows the formation of a closure-like domain in this bilayer system, with the penetration of the R-domain in the T-layer and T-domain in the R-layer. The wedge domain is also shown in Figure 5 (c), where the polarization along the other orthogonal plane is plotted. Overall, the coupling of the PZT (T) and the PZT (R) layers has given rise to two interesting features at the interface. First, the formation of a rotated phase to decrease the polar discontinuity and minimize the bound charges. Second, penetrating $a$-domains with wedge-like shapes, which could be more mobile upon the application of external stimuli. 


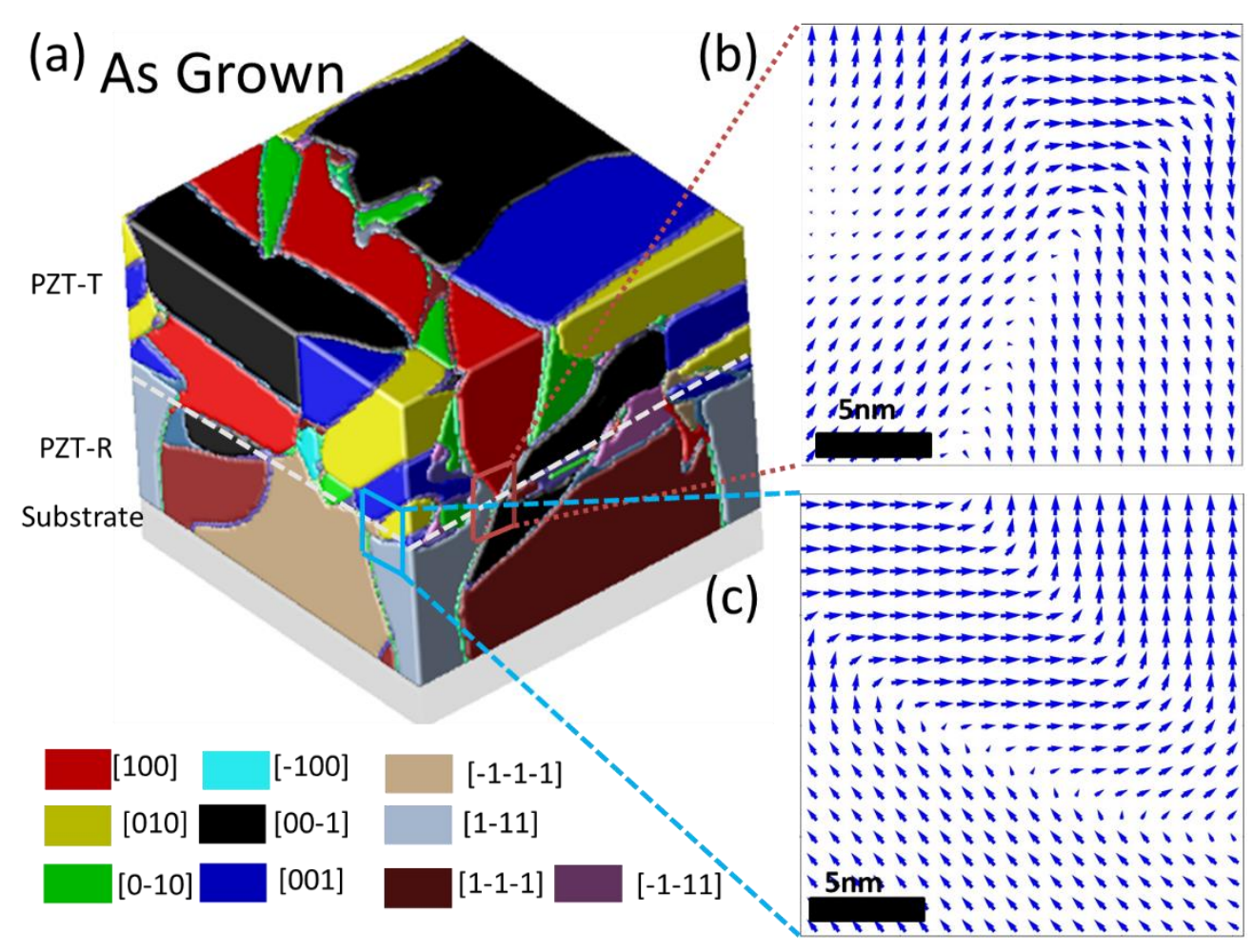

Figure 5. (a) Phase field simulated domain structure of the as-grown $T / R$ bilayered film. (b) Local polarization profile of the region marked by a red rectangular box in (a) shows the formation of closure-like domains, with the penetration of the R-domain in the T-layer and Tdomain in the R-layer. (c) Local polarization profile of the region marked by a blue rectangular box in which a wedge domain is shown.

The domain structure after switching is given in Figure 6 (a). A $3 \mathrm{~V}$ bias is applied through the electrode on the film top. It is clearly shown that the $c+$ domain has been completely switched due to the applied negative electric field. Whilst the large $a$-domains do not switch, small $a$-domains are removed, with the nucleation and growth of new, large $a$-domains, forming a cross-hatch pattern. The simple R-twin domain with only 71-degree walls is shown in the bottom R-layer. Figure 6 (b) shows the polar plot near the interface in the same location as Figure 5 (b). It can be seen that upon the application of an electric field, the closure domain disappears due to the ferroelastic domain wall motion and the polarization switching. However, 
the penetration of R-domains and T-domains still exist. Figure 6 (c) shows the same area as Figure 5 (c), in comparison, we see that the switching of polarization in the R-domain from upward to downward introduces a tail-to-tail domain configuration. As a result, the system adopts two paths to minimize the charged domain wall. Firstly, a new $a_{1} / c$ domain is formed by the movement of the large $a_{1}$-domain, and secondly, the switching of the $a_{2}{ }^{+}$to $a_{2}{ }^{-}$domain. This confirms that an application of an electric field leads to a dramatic rearrangement of the polarization jigsaw, and one that can only be stabilized by motion of the $a$-domains.

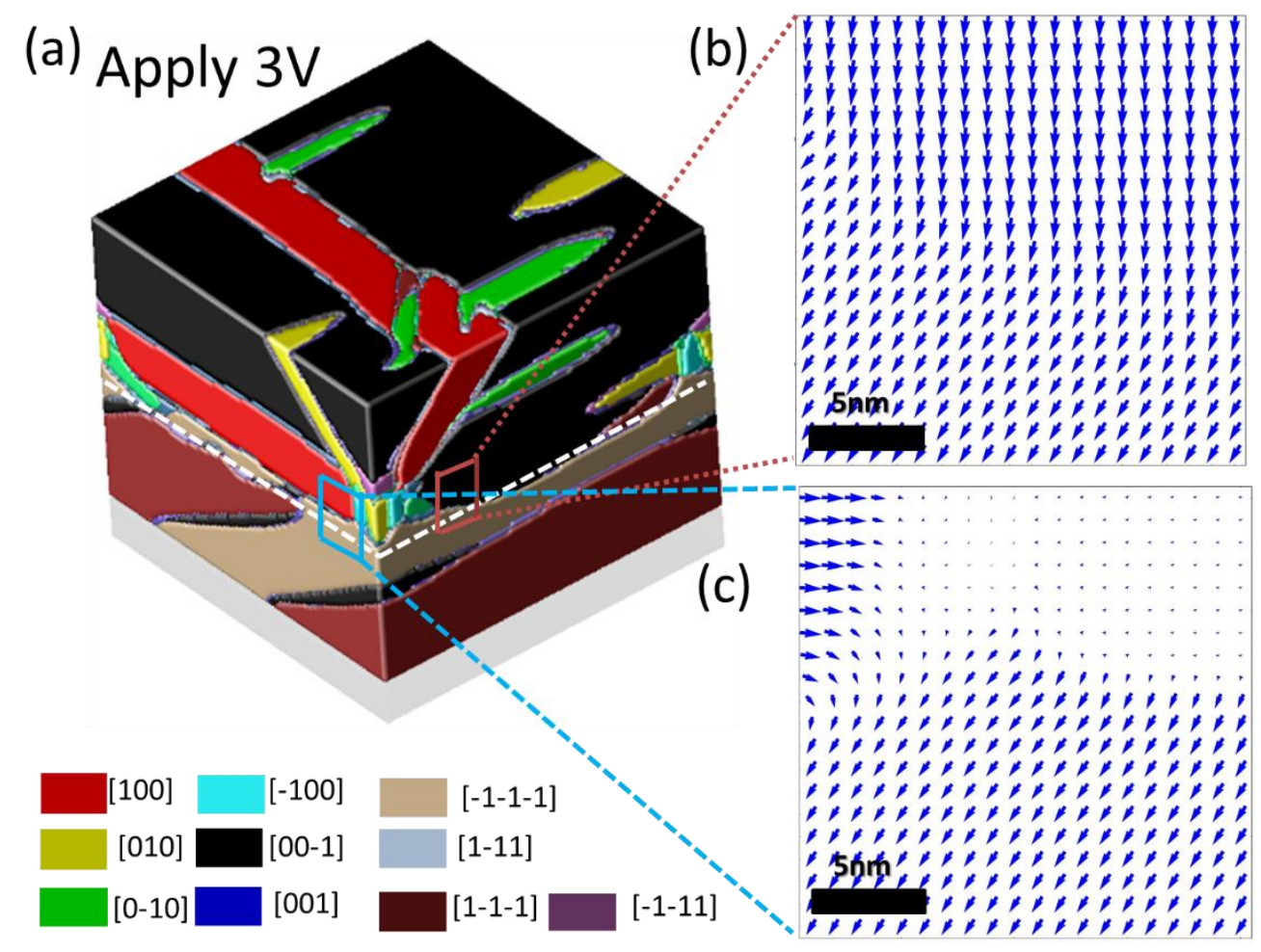

Figure 6. (a) Phase field simulated domain structure of the $T / R$ bilayered thin films after applying an electric field of $3 \mathrm{~V}$. (b) Local polarization plot of the region marked by a red rectangular box in (a) shows that the closure domain disappears, whilst the penetration of the R-domain in the T-layer still exists. (c) Local polarization plot of the region marked by a blue rectangular box in which a wedge domain is shown. 
In summary, we shed some insight into why multilayer ferroelectric thin films have been recently found to possess high ferroelastic domain wall mobility compared single-layer counter parts. The key to the process lies in the understanding of the complex domain arrangements at the vicinity of the ferroelastic domain wall. Using epitaxial $100 \mathrm{~nm}$ thick (001) tetragonal (T) $\mathrm{PbZr}_{\mathrm{x}} \mathrm{Ti}_{1-\mathrm{x}} \mathrm{O}_{3}(\mathrm{PZT}) /$ rhombohedral (R) $\mathrm{PbZr}_{\mathrm{x}} \mathrm{Ti}_{1-\mathrm{x}} \mathrm{O}_{3}(\mathrm{PZT})$ epitaxial bilayer heterostructures as our model test system, we map the atomic-scale polarization displacement and orientation at/near the composition breaking interface which includes a ferroelastic domain wall. We propose that the enormous local strain gradient in the vicinity of the domain wall is large enough to not only locally rotate the polarizations in the $\mathrm{T}$ and $\mathrm{R}$ layers, but also create complex domain topologies. Phase field simulations find that this complex domain arrangement is driven by minimization of the excessive elastic and electrostatic energies due to the strain gradient near/at the interface. As a result, any external stimuli that rearranges one component of the polarization necessarily results in the large-scale domain rearrangement including the movement of $a$-domains, thus leading ferroelastic domain wall mobility. We do appreciate that in this model we did not consider the effect of local screening charges, which are shown to be critical by previous STEM-based investigations. ${ }^{71}$ Secondly how local charges contribute to the switching mechanisms of ferroelastic domains in multilayers still remains unresolved. However, given that one key factor has now been identified, the next series of investigations will be focused on mapping electronic-structure particularly for point defect motion in the vicinity of the ferroelastic domain wall.

\section{METHODS}

\section{Film fabrication:}

$100 \mathrm{~nm}$ thick $\mathrm{PbZr}_{0.30} \mathrm{Ti}_{0.70} \mathrm{O}_{3}(\mathrm{PZT}(\mathrm{T})) / 100 \mathrm{~nm}$ thick $\mathrm{PbZr}_{0.55} \mathrm{Ti}_{0.45} \mathrm{O}_{3}(\mathrm{PZT}(\mathrm{R}))$ epitaxial thin films were fabricated by pulsed laser deposition (PLD) on a $\mathrm{SrTiO}_{3}$ (STO) (001) substrate with a $\mathrm{La}_{0.67} \mathrm{Sr}_{0.33} \mathrm{MnO}_{3}(\mathrm{LSMO})(20 \mathrm{~nm})$ buffer layer. Details can be found in the reference. ${ }^{13}$ 


\section{TEM/STEM sample preparation and observation:}

Cross-sectional transmission electron microscope (TEM) specimens were prepared by the standard tripod wedge polishing technique, followed by argon ion thinning at 1-3 $\mathrm{kV}$ using a precision ion polishing system (PIPS). Cross-sectional TEM imaging was performed using a Philips CM200 TEM with an accelerating voltage of $200 \mathrm{kV}$. Atomic-scale imaging was undertaken using an aberration-corrected scanning transmission electron microscope (Cscorrected STEM) (Hitachi HD-2700) installed at Brookhaven National Laboratory in New York. ${ }^{72}$ This is equipped with a cold-field electron source and a high-resolution electron energy-loss spectrometer (EELS) (Gatan, Enfina detector). Atomic resolution annular dark field (ADF) STEM images were recorded at an accelerating voltage of $200 \mathrm{kV}$. Therein, the HAADF-STEM imaging comprised collection angles of 114-608 mrad. In order to improve the signal-to-noise ratio, while minimizing the sample damage, we used a beam current of 120 pA and a pixel dwell time of $16.2 \mu$ sec to collect all the data. To identify the interface between the two PZT layers, electron energy loss spectroscopy (EELS) was performed by a parallel EELS detector with an energy resolution of $0.35 \mathrm{eV}$. All STEM figures were processed for drift and noise correction. The noise correction procedure used was through the standard Fourier filtering available in Digital MicrographTM software (DM, Gatan Inc). ${ }^{73}$

\section{Atomic column position identification:}

The atomic positions were determined by a simultaneous fitting of the individual atomic columns to two dimensional Gaussian peaks using a custom code developed by "MatLab R2013a" software packages. Polarization displacements of the $\mathrm{Zr}$ /Ti cations in HAADF-STEM images were measured relative to the centre of the surrounding $\mathrm{Pb}$ cations. Details can be found in the Section S5.

\section{Phase field simulation methods:}


For the phase field simulations, a three-dimensional mesh of $128 \times 128 \times 128$ is constructed, with each grid representing $1 \mathrm{~nm}$, where the thickness direction is composed of $15 \mathrm{~nm}$ of substrate, $100 \mathrm{~nm}$ of film (50 nm of PZT (T) and $50 \mathrm{~nm}$ of PZT (R) layers) and $13 \mathrm{~nm}$ of air. Periodic boundary conditions are applied in the planar directions, while a superposition scheme is used in the other dimension. Complete relaxation of strain is considered for both layers, which is verified by experimental observations. We limit the threshold for free carriers to the value required to screen the bound charge due to the polarization gradient, i.e., $\rho=-\nabla \vec{P}$. Random noise is added as the initial state to model the thermal fluctuations during the growth procedure. An iterative perturbation method is used to consider the differences in elastic constants for the T-layer and the R-layer. ${ }^{74-75}$

\section{AUTHOR INFORMATION}

\section{Corresponding Author}

*nagarajan@unsw.edu.au

\section{Author Contributions}

The manuscript was written through contributions of all authors. All authors have given approval to the final version of the manuscript. $\ddagger$ Z.J.H. and H.L.X. contributed equally. H.L.X. and D.S. assisted the Cs-STEM observations. H.L.X. and G.H. conducted the polarization measurements. L.-Q.C. and Z.J.H. carried out the phase field simulation analysis.

\section{Funding Sources}

The research at the University of New South Wales was supported by an ARC Discovery Project. STEM work made use of resources of the Center for Functional Nanomaterials, which is a U.S. DOE Office of Science Facility, at Brookhaven National Laboratory under Contract No. DE-SC0012704. Phase field simulation work is supported by U.S. Department of Energy, 
Office of Basic Energy Sciences, Division of Materials Sciences and Engineering under Award FG02-07ER46417 (LQC). Z.J.H. acknowledges the support by NSF-MRSEC grant number DMR-1420620 and NSF-MWN grant number DMR-1210588.

\section{ACKNOWLEDGMENTS}

The authors would like to thank E. Huang for providing samples. The authors wish to acknowledge J. Halverson for useful discussion and help on tetragonality measurements. NV acknowledges detailed discussions with L.W. Martin.

Supporting Information. Supporting information available:

Full details of polydomain identification using GPA, STEM-based atomic displacement mapping, location of the R-T interface using EELS mapping and identification of polarization displacement vectors inside the individual $\mathrm{T}$ and R-layer. This material is available free of charge via the Internet at http://pubs.acs.org.

\section{REFERENCES}

1. Bousquet, E.; Dawber, M.; Stucki, N.; Lichtensteiger, C.; Hermet, P.; Gariglio, S.;

Triscone, J.-M.; Ghosez, P., Improper Ferroelectricity in Perovskite Oxide Artificial Superlattices. Nature 2008, 452, 732-736.

2. Haeni, J. H.; Irvin, P.; Chang, W.; Uecker, R.; Reiche, P.; Li, Y. L.; Choudhury, S.; Tian, W.; Hawley, M. E.; Craigo, B.; Tagantsev, A. L.; Pan, X. Q.; Streiffer S. L.; Chen, L. Q.; Kirchoefer, S. W.; Levy, J.; Schlom, D. G., Room-Temperature Ferroelectricity in Strained $\mathrm{SrTiO}_{3}$. Nature 2004, 430, 758-761. 
3. Choi, K. J.; Biegalski, M.; Li, Y. L.; Sharan, A.; Schubert, J.; Uecker, R.; Reiche, P.; Chen, Y. B.; Pan, X. Q.; Gopalan, V.; Chen, L.-Q.; Schlom, D. G.; Eom, C. B., Enhancement of Ferroelectricity in Strained $\mathrm{BaTiO}_{3}$ Thin Films. Science 2004, 306, 1005-1009.

4. Garcia, V.; Bibes, M.; Bocher, L.; Valencia, S.; Kronast, F.; Crassous, A.; Moya, X.; Enouz-Vedrenne, S.; Gloter, A.; Imhoff, D.; Deranlot, C.; Mathur, N. D.; Fusil, S.; Bouzehouane, K.; Barthelemy, A., Ferroelectric Control of Spin Polarization. Science 2010, 327, 1106-1110.

5. Yin, Y. W.; Burton, J. D.; Kim, Y. M.; Borisevich, A. Y.; Pennycook, S. J.; Yang, S. M.; Noh, T. W.; Gruverman, A.; Li, X. G.; Tsymbal, E. Y.; Li, Q., Enhanced Tunnelling Electroresistance Effect Due to a Ferroelectrically Induced Phase Transition at a Magnetic Complex Oxide Interface. Nat. Mater. 2013, 12, 397-402.

6. Mangalam, R. V. K.; Karthik, J.; Damodaran, A. R.; Agar, J. C.; Martin, L. W., Unexpected Crystal and Domain Structures and Properties in Compositionally Graded $\mathrm{PbZr}_{1-}$ ${ }_{x} \mathrm{Ti}_{\mathrm{x}} \mathrm{O}_{3}$ Thin Films. Adv. Mater. 2013, 25, 1761-1767.

7. Okatan, M. B.; Roytburd, A. L.; Mantese, J. V.; Alpay, S. P., Domain Engineering in Compositionally Graded Ferroelectric Films for Enhanced Dielectric Response and Tunability. J. Appl. Phys. 2009, 105, 114106.

8. Cole, M. W.; Ngo, E.; Hirsch, S.; Okatan, M. B.; Alpay, S. P., Dielectric Properties of MgO-Doped Compositionally Graded Multilayer Barium Strontium Titanate Films. Appl. Phys. Lett. 2008, 92, 072906.

9. Mangalam, R. V. K.; Agar, J. C.; Damodaran, A. R.; Karthik, J.; Martin, L. W., Improved Pyroelectric Figures of Merit in Compositionally Graded $\mathrm{PbZr}_{1-\mathrm{x}} \mathrm{Ti}_{\mathrm{x}} \mathrm{O}_{3}$ Thin Films. ACS Appl. Mater. Interfaces 2013, 5, 13235-13241. 
10. Bastani, Y.; Bassiri-Gharb, N., Enhanced Dielectric and Piezoelectric Response in PZT Superlattice-Like Films by Leveraging Spontaneous Zr/Ti Gradient Formation. Acta Mater. 2012, 60, 1346-1352.

11. Okatan, M. B.; Roytburd, A. L.; Mantese, J. V.; Alpay, S. P., Domain Engineering in Compositionally Graded Ferroelectric Films for Enhanced Dielectric Response and Tunability. J. Appl. Phys. 2009, 105, 114106.

12. Nath, R.; Zhong, S.; Alpay, S. P.; Huey, B. D.; Cole, M. W., Enhanced Piezoelectric Response from Barium Strontium Titanate Multilayer Films. Appl. Phys. Lett. 2008, 92, 012916.

13. Huang, H.-H.; Zhang, Q.; Huang, E.; Maran, R.; Sakata, O.; Ehara, Y.; Shiraishi, T.; Funakubo, H.; Munroe, P.; Valanoor, N., Epitaxial $\mathrm{PbZr}_{\mathrm{x}} \mathrm{Ti}_{1-\mathrm{x}} \mathrm{O}_{3}$ Ferroelectric Bilayers with Giant Electromechanical Properties. Adv. Mater. Interfaces 2015, 2, 1500075.

14. Agar, J. C.; Damodaran, A. R.; Okatan, M. B.; Kacher, J.; Gammer, C.; Vasudevan, R. K.; Pandya, S.; Dedon, L. R.; Mangalam, R. V. K.; Velarde, G. A.; Jesse, S.; Balke, N.; Minor, A. M.; Kalinin, S. V.; Martin, L. W., Highly Mobile Ferroelastic Domain Walls in Compositionally Graded Ferroelectric Thin Films. Nat. Mater. 2016, 15, 549-556.

15. Bao, D.; Yao, X.; Zhang, L., Dielectric Enhancement and Ferroelectric Anomaly of Compositionally Graded ( $\mathrm{Pb}, \mathrm{Ca}) \mathrm{TiO}_{3}$ Thin Films Derived by a Modified Sol-Gel Technique. Appl. Phys. Lett. 2000, 76, 2779-2781.

16. Anton, S. R.; Sodano, H. A., A Review of Power Harvesting Using Piezoelectric Materials (2003-2006). Smart Mater. Struct. 2007, 16, R1.

17. Chen, J.; Panda, R. In Review: Commercialization of Piezoelectric Single Crystals for Medical Imaging Applications, IEEE Ultrason. Symp., 2005; pp 235-240. 
18. Ko, H.-P.; Jeong, H.; Koc, B., Piezoelectric Actuator for Mobile Auto Focus Camera Applications. J. Electroceram. 2008, 23, 530-535.

19. Anbusathaiah, V.; Kan, D.; Kartawidjaja, F. C.; Mahjoub, R.; Arredondo, M. A.; Wicks, S.; Takeuchi, I.; Wang, J.; Nagarajan, V., Labile Ferroelastic Nanodomains in Bilayered Ferroelectric Thin Films. Adv. Mater. 2009, 21, 3497-3502.

20. Crassous, A.; Sluka, T.; Tagantsev, A. K.; Setter, N., Polarization Charge as a Reconfigurable Quasi-Dopant in Ferroelectric Thin Films. Nat. Nanotechnol. 2015, 10, 614618.

21. Bednyakov, P. S.; Sluka, T.; Tagantsev, A. K.; Damjanovic, D.; Setter, N., Formation of Charged Ferroelectric Domain Walls with Controlled Periodicity. Sci. Rep. 2015, 5, 15819.

22. Sluka, T.; Tagantsev, A. K.; Damjanovic, D.; Gureev, M.; Setter, N., Enhanced Electromechanical Response of Ferroelectrics Due to Charged Domain Walls. Nat. Commun. 2012, 3, 748 .

23. Feigl, L.; Yudin, P.; Stolichnov, I.; Sluka, T.; Shapovalov, K.; Mtebwa, M.; Sandu, C. S.; Wei, X.-K.; Tagantsev, A. K.; Setter, N., Controlled Stripes of Ultrafine Ferroelectric Domains. Nat. Commun. 2014, 5, 4677.

24. Shvartsman, V. V.; Pertsev, N. A.; Herrero, J. M.; Zaldo, C.; Kholkin, A. L., Nonlinear Local Piezoelectric Deformation in Ferroelectric Thin Films Studied by Scanning Force Microscopy. J. Appl. Phys. 2005, 97, 104105.

25. Griggio, F.; Jesse, S.; Kumar, A.; Ovchinnikov, O.; Kim, H.; Jackson, T. N.; Damjanovic, D.; Kalinin, S. V.; Trolier-McKinstry, S., Substrate Clamping Effects on Irreversible Domain Wall Dynamics in Lead Zirconate Titanate Thin Films. Phys. Rev. Lett. 2012, 108, 157604. 
26. Xu, F.; Trolier-McKinstry, S.; Ren, W.; Xu, B.; Xie, Z.-L.; Hemker, K. J., Domain Wall Motion and Its Contribution to the Dielectric and Piezoelectric Properties of Lead Zirconate Titanate Films. J. Appl. Phys. 2001, 89, 1336-1348.

27. Mahjoub, R.; Anbusathaiah, V.; Alpay, S. P.; Nagarajan, V., Ferroelastic Domains in Bilayered Ferroelectric Thin Films. J. Appl. Phys. 2008, 104, 124103.

28. Xue, F.; Wang, J. J.; Sheng, G.; Huang, E.; Cao, Y.; Huang, H. H.; Munroe, P.; Mahjoub, R.; Li, Y. L.; Nagarajan, V.; Chen, L. Q., Phase Field Simulations of Ferroelectrics Domain Structures in $\mathrm{PbZr}_{\mathrm{x}} \mathrm{Ti}_{1-\mathrm{x}} \mathrm{O}_{3}$ Bilayers. Acta Mater. 2013, 61, 2909-2918.

29. Bhaskar, U. K.; Banerjee, N.; Abdollahi, A.; Wang, Z.; Schlom, D. G.; Rijnders, G.; Catalan, G., A Flexoelectric Microelectromechanical System on Silicon. Nat. Nanotechnol. 2016, 11, 263-266.

30. Bhaskar, U. K.; Banerjee, N.; Abdollahi, A.; Solanas, E.; Rijnders, G.; Catalan, G., Flexoelectric Mems: Towards an Electromechanical Strain Diode. Nanoscale 2016, 8, 12931298.

31. Stemmer, S.; Streiffer, S. K.; Ernst, F.; Rüuhle, M., Atomistic Structure of $90^{\circ}$ Domain Walls in Ferroelectric $\mathrm{PbTiO}_{3}$ Thin Films. Philos. Mag. A 1995, 71, 713-724.

32. Shilo, D.; Ravichandran, G.; Bhattacharya, K., Investigation of Twin-Wall Structure at the Nanometre Scale Using Atomic Force Microscopy. Nat. Mater. 2004, 3, 453-457.

33. Chandrasekaran, A.; Wei, X.-K.; Feigl, L.; Damjanovic, D.; Setter, N.; Marzari, N., Asymmetric Structure of $90^{\circ}$ Domain Walls and Interactions with Defects in $\mathrm{PbTiO}_{3}$. Phys Rev. B 2016, 93, 144102. 
34. Jia, C.-L.; Mi, S.-B.; Urban, K.; Vrejoiu, I.; Alexe, M.; Hesse, D., Atomic-Scale Study of Electric Dipoles near Charged and Uncharged Domain Walls in Ferroelectric Films. Nat. Mater. 2008, 7, 57-61.

35. Lubk, A.; Rossell, M. D.; Seidel, J.; Chu, Y. H.; Ramesh, R.; Hÿtch, M. J.; Snoeck, E., Electromechanical Coupling among Edge Dislocations, Domain Walls, and Nanodomains in $\mathrm{BiFeO}_{3}$ Revealed by Unit-Cell-Wise Strain and Polarization Maps. Nano Lett. 2013, 13, 1410 1415.

36. Rossell, M. D.; Erni, R.; Prange, M. P.; Idrobo, J. C.; Luo, W.; Zeches, R. J.; Pantelides, S. T.; Ramesh, R., Atomic Structure of Highly Strained $\mathrm{BiFeO}_{3}$ Thin Films. Phys. Rev. Lett. 2012, 108, 047601.

37. Tang, Y. L.; Zhu, Y. L.; Ma, X. L.; Borisevich, A. Y.; Morozovska, A. N.; Eliseev, E. A.; Wang, W. Y.; Wang, Y. J.; Xu, Y. B.; Zhang, Z. D.; Pennycook, S. J., Observation of a Periodic Array of Flux-Closure Quadrants in Strained Ferroelectric $\mathrm{PbTiO}_{3}$ Films. Science 2015, $348,547-551$.

38. Tang, Y. L.; Zhu, Y. L.; Wang, Y. J.; Wang, W. Y.; Xu, Y. B.; Ren, W. J.; Zhang, Z. D.; Ma, X. L., Atomic-Scale Mapping of Dipole Frustration at $90^{\circ}$ Charged Domain Walls in Ferroelectric $\mathrm{PbTiO}_{3}$ Films. Sci. Rep. 2014, 4, 4115.

39. Zubko, P.; Jecklin, N.; Torres-Pardo, A.; Aguado-Puente, P.; Gloter, A.; Lichtensteiger, C.; Junquera, J.; Stéphan, O.; Triscone, J. M., Electrostatic Coupling and Local Structural Distortions at Interfaces in Ferroelectric/Paraelectric Superlattices. Nano Lett. 2012, 12, 28462851. 
40. Jia, C.-L.; Nagarajan, V.; He, J.-Q.; Houben, L.; Zhao, T.; Ramesh, R.; Urban, K.; Waser, R., Unit-Cell Scale Mapping of Ferroelectricity and Tetragonality in Epitaxial Ultrathin Ferroelectric Films. Nat. Mater. 2007, 6, 64-69.

41. Nelson, C. T.; Gao, P.; Jokisaari, J. R.; Heikes, C.; Adamo, C.; Melville, A.; Baek, S.H.; Folkman, C. M.; Winchester, B.; Gu, Y.; Liu, Y.; Zhang, K., Wang, E.; Li, J.; Chen, L.-Q.; Eom, C.-B.; Schlom, D. G.; Pan, X., Domain Dynamics During Ferroelectric Switching. Science 2011, 334, 968-971.

42. Gao, P.; Nelson, C. T.; Jokisaari, J. R.; Baek, S.-H.; Bark, C. W.; Zhang, Y.; Wang, E.; Schlom, D. G.; Eom, C.-B.; Pan, X., Revealing the Role of Defects in Ferroelectric Switching with Atomic Resolution. Nat. Commun. 2011, 2, 591.

43. Chang, H.; Kalinin, S. V.; Yang, S.; Yu, P.; Bhattacharya, S.; Wu, P. P.; Balke, N.; Jesse, S.; Chen, L. Q.; Ramesh, R.; Pennycook, S. J.; Borisevich, A. Y., Watching Domains Grow: In-Situ Studies of Polarization Switching by Combined Scanning Probe and Scanning Transmission Electron Microscopy. J. Appl. Phys. 2011, 110, 052014.

44. Muller, D. A.; Nakagawa, N.; Ohtomo, A.; Grazul, J. L.; Hwang, H. Y., Atomic-Scale Imaging of Nanoengineered Oxygen Vacancy Profiles in $\mathrm{SrTiO}_{3}$. Nature 2004, 430, 657-661.

45. Muller, D. A.; Kourkoutis, L. F.; Murfitt, M.; Song, J. H.; Hwang, H. Y.; Silcox, J.; Dellby, N.; Krivanek, O. L., Atomic-Scale Chemical Imaging of Composition and Bonding by Aberration-Corrected Microscopy. Science 2008, 319, 1073-1076.

46. Spurgeon, S. R.; Balachandran, P. V.; Kepaptsoglou, D. M.; Damodaran, A. R.; Karthik, J.; Nejati, S.; Jones, L.; Ambaye, H.; Lauter, V.; Ramasse, Q. M.; Lau, K. K. S.; Martin, L. W.; Rondinelli, J. M.; Taheri, M. L., Polarization Screening-Induced Magnetic Phase Gradients at Complex Oxide Interfaces. Nat. Commun. 2015, 6, 6735. 
47. Catalan, G.; Lubk, A.; Vlooswijk, A. H. G.; Snoeck, E.; Magen, C.; Janssens, A.; Rispens, G.; Rijnders, G.; Blank, D. H. A.; Noheda, B., Flexoelectric Rotation of Polarization in Ferroelectric Thin Films. Nat. Mater. 2011, 10, 963-967.

48. Mitsui T.; Nakamura E.; Shiozaki Y., Landolt-Börnstein, Numerical

Data and Functional Relationships in Science and Technology. Springer: Berlin, 1981; Vol. 16.

49. Hovestreydt, E., On the Atomic Scattering Factor for $\mathrm{O}_{2}$. Acta Cryst. A 1983, 39, 268269.

50. Jia, C.-L.; Urban, K. W.; Alexe, M.; Hesse, D.; Vrejoiu, I., Direct Observation of Continuous Electric Dipole Rotation in Flux-Closure Domains in Ferroelectric $\mathrm{Pb}(\mathrm{Zr}, \mathrm{Ti}) \mathrm{O}_{3}$. Science 2011, 331, 1420-1423.

51. Streiffer, S. K.; Parker, C. B.; Romanov, A. E.; Lefevre, M. J.; Zhao, L.; Speck, J. S.; Pompe, W.; Foster, C. M.; Bai, G. R., Domain Patterns in Epitaxial Rhombohedral Ferroelectric Films. I. Geometry and Experiments. J. Appl. Phys. 1998, 83, 2742-2753.

52. Nelson, C. T.; Winchester, B.; Zhang, Y.; Kim, S.-J.; Melville, A.; Adamo, C.; Folkman, C. M.; Baek, S.-H.; Eom, C.-B.; Schlom, D. G.; Chen, L.-Q.; Pan, X., Spontaneous Vortex Nanodomain Arrays at Ferroelectric Heterointerfaces. Nano Lett. 2011, 11, 828-834.

53. Ren, X., Large Electric-Field-Induced Strain in Ferroelectric Crystals by Point-DefectMediated Reversible Domain Switching. Nat. Mater. 2004, 3, 91-94.

54. Liu, Y.; Tang, Y.-L.; Zhu, Y.-L.; Wang, W.-Y.; Ma, X.-L., Ferroelectric Films: Spatial Coupling of Ferroelectric Domain Walls and Crystallographic Defects in the $\mathrm{PbTiO}_{3}$ Films, Adv. Mater. Interfaces 2016, 3, 1600342. 
55. Hannes, L.; Michael, L., Electron Holography—Basics and Applications. Rep. Prog. Phys. 2008, 71, 016102.

56. Jia, C.-L.; Mi, S.-B.; Urban, K.; Vrejoiu, I.; Alexe, M.; Hesse, D., Atomic-Scale Study of Electric Dipoles near Charged and Uncharged Domain Walls in Ferroelectric Films. Nat Mater 2008, 7, 57-61.

57. Houchmandzadeh, B.; Lajzerowicz, J.; Salje, E., Order Parameter Coupling and Chirality of Domain Walls. J. Phys. Cond. Matter 1991, 3, 5163.

58. Srolovitz, D. J.; Scott, J. F., Clock-Model Description of Incommensurate Ferroelectric Films and of Nematic-Liquid-Crystal Films. Phys. Rev. B 1986, 34, 1815-1819.

59. Gruverman, A.; Wu, D.; Fan, H. J.; Vrejoiu, I.; Alexe, M.; Harrison, R. J.; Scott, J. F., Vortex Ferroelectric Domains. J. Phys. Cond. Matter 2008, 20, 342201.

60. McQuaid, R. G. P.; McGilly, L. J.; Sharma, P.; Gruverman, A.; Gregg, J. M., Mesoscale Flux-Closure Domain Formation in Single-Crystal BaTiO3. Nat. Commun. 2011, 2, 404.

61. Ivry, Y.; Chu, D. P.; Scott, J. F.; Durkan, C., Flux Closure Vortexlike Domain Structures in Ferroelectric Thin Films. Phys. Rev. Lett. 2010, 104, 207602.

62. Wohlhuter, P.; Bryan, M. T.; Warnicke, P.; Gliga, S.; Stevenson, S. E.; Heldt, G.; Saharan, L.; Suszka, A. K.; Moutafis, C.; Chopdekar, R. V.; Raabe, J.; Thomson, T.; Hrkac, G.; Heyderman, L. J., Nanoscale Switch for Vortex Polarization Mediated by Bloch Core Formation in Magnetic Hybrid Systems. Nat. Commun. 2015, 6, 7836.

63. Schilling, A.; Byrne, D.; Catalan, G.; Webber, K. G.; Genenko, Y. A.; Wu, G. S.; Scott, J. F.; Gregg, J. M., Domains in Ferroelectric Nanodots. Nano Lett. 2009, 9, 3359-3364. 
64. Zubko, P.; Catalan, G.; Buckley, A.; Welche, P. R. L.; Scott, J. F., Strain-GradientInduced Polarization in $\mathrm{SrTiO}_{3}$ Single Crystals. Phys. Rev. Lett. 2007, 99, 167601.

65. Lu, H.; Bark, C.-W.; Esque de los Ojos, D.; Alcala, J.; Eom, C. B.; Catalan, G.; Gruverman, A., Mechanical Writing of Ferroelectric Polarization. Science 2012, 336, 59-61.

66. Zubko, P.; Catalan, G.; Tagantsev, A. K., Flexoelectric Effect in Solids. Annu. Rev. Mater. Res. 2013, 43, 387-421.

67. Catalan, G.; Lubk, A.; Vlooswijk, A. H. G.; Snoeck, E.; Magen, C.; Janssens, A.; Rispens, G.; Rijnders, G.; Blank, D. H. A.; Noheda, B., Flexoelectric Rotation of Polarization in Ferroelectric Thin Films. Nat. Mater. 2011, 10, 963-967.

68. Houben, L.; Thust, A.; Urban, K., Atomic-Precision Determination of the Reconstruction of a Tilt Boundary in by Aberration Corrected Hrtem. Ultramicroscopy 2006, $106,200-214$.

69. Liu, G.; Zhang, Q.; Huang, H.-H.; Munroe, P.; Nagarajan, V.; Simons, H.; Hong, Z.; Chen, L.-Q., Reversible Polarization Rotation in Epitaxial Ferroelectric Bilayers. Adv. Mater. Interfaces 2016, 1600444.

70. Li, Y. L.; Hu, S. Y.; Liu, Z. K.; Chen, L. Q., Effect of Substrate Constraint on the Stability and Evolution of Ferroelectric Domain Structures in Thin Films. Acta Mater. 2002, $50,395-411$.

71. Kim, Y.-M.; Kumar, A.; Hatt, A.; Morozovska, A. N.; Tselev, A.; Biegalski, M. D.; Ivanov, I.; Eliseev, E. A.; Pennycook, S. J.; Rondinelli, J. M.; Kalinin, S. V.; Borisevich, A. Y., Interplay of Octahedral Tilts and Polar Order in $\mathrm{BiFeO}_{3}$ Films. Adv. Mater. 2013, 25, 24972504. 
72. Inada, H.; Wu, L.; Wall, J.; Su, D.; Zhu, Y., Performance and Image Analysis of the Aberration-Corrected Hitachi HD-2700C STEM. J. Electron Microsc. 2009, 58, 111-122.

73. Williams, D. B.; Carter, C. B., Transmission Electron Microscopy: A Textbook for Materials Science. Springer: 2009; pp 551.

74. Wang, J. J.; Ma, X. Q.; Li, Q.; Britson, J.; Chen, L.-Q., Phase Transitions and Domain Structures of Ferroelectric Nanoparticles: Phase Field Model Incorporating Strong Elastic and Dielectric Inhomogeneity. Acta Mater. 2013, 61, 7591-7603.

75. Yadav, A. K.; Nelson, C. T.; Hsu, S. L.; Hong, Z.; Clarkson, J. D.; Schlepüetz, C. M.; Damodaran, A. R.; Shafer, P.; Arenholz, E.; Dedon, L. R.; Chen, D., Vishwanath, A.; Minor, A. M.; Chen, L. Q.; Scott, J. F.; Martin, L. W.; Ramesh, R., Observation of Polar Vortices in Oxide Superlattices. Nature 2016, 530, 198-201. 
For Table of Contents

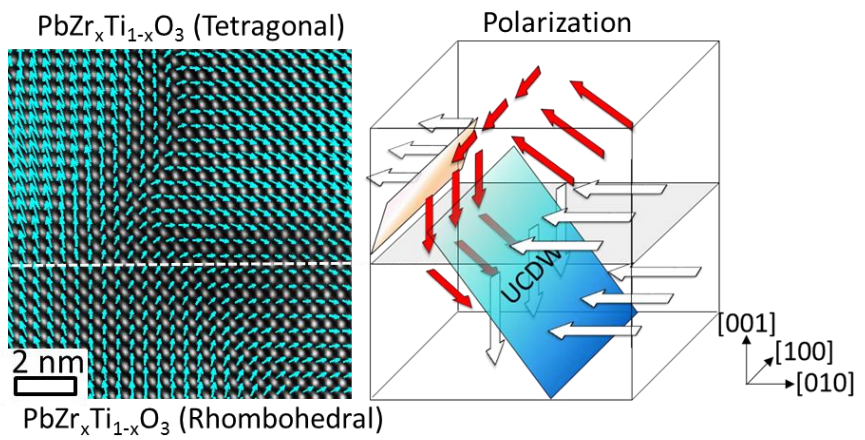

